\title{
Small structures on a big scale
}

DNA origami, the bottom-up self-assembly of designed 3-D nanostructures, is a promising new method for producing complex materials and delivery vehicles for therapeutic agents. But the process for developing DNA origami structures is expensive and time-consuming, limiting the batch sizes of final products to just micrograms. In a recent paper in Nature, a group in Germany led by Hendrik Dietz has developed an efficient workflow for mass production of DNA origami using standard laboratory methods that won't break the bank (Nature 552, 84-87; 2017).

DNA origami structures are assembled from a long single-stranded scaffolds, which are held in place using shorter 'staple strands'. While long scaffolds can be easily generated using standard bacteriophage methods in the lab, producing short staple strands requires much more expensive synthesis processes. To solve this problem, Dietz and colleagues incorporated small self-cleaving DNA cassettes (called DNAzymes) into longer stretches of DNA that can also be easily produced en mass by bacteriophages. These cassettes, when activated by addition of zinc, automatically cleave themselves out, producing shorter staple strands that combine with scaffolds produced in parallel to form origami DNA structures.
Using 4 different designed structures ( 2 novel, and 2 previously generated in other reports), the team demonstrated their protocol's generalizability. Likewise, the group showed their ability to produce large quantities of DNA origami structures $(\sim 167 \mathrm{mg})$ using a laboratory-scale stirred-tank bioreactor. The team estimated the cost of their production protocol to be $€ 23$ per milligram, but note that with even more efficient and standard industry methods for bacteriophage production, the cost could drop to as low as $€ 0.18$ per milligram.

Dustin M. Graham 